\title{
Modified Early DBA Algorithm for Reducing Packet Delay and Waiting Time of Unstable ONUs in PON
}

\author{
Bipasha Kundu, Monir Hossen, Sujit Basu, Md. Imamul Arefeen \\ Department of Electronics and Communication Engineering \\ Khulna University of Engineering \& Technology \\ Khulna, Bangladesh
}

\begin{abstract}
Passive optical network (PON) is a highly dynamic access network that effectively converge several service providers. In a time cycle, some optical network units (ONUs) of PON may miss the usual polling instant called unstable ONUs. According to the existing early dynamic bandwidth allocation (E-DBA) algorithm, once an ONU miss the usual polling instant it needs to be waited until the end of transmissions from the entire stable ONUs. So the unstable ONUs suffers from more waiting time than the stable ONUs. This paper proposes a new DBA algorithm called modified E-DBA (ME-DBA) algorithm, for reduction of waiting times of the unstable ONUs. In the proposed algorithm, total ONUs are divided into several small groups. The data packets from all the stable ONUs of a group are transmitted $1^{\text {st }}$ and then the data packets from the unstable ONUs of that group are transmitted. We have analyzed and compared the performance of the proposed ME-DBA algorithm with the existing E-DBA algorithm in terms of the delay and waiting time of the unstable ONUs by simulations. The simulation results show that the proposed algorithm provides lower packet delay and waiting time for the unstable ONUs than that of the existing E-DBA algorithm.
\end{abstract}

Keywords: $P O N, D B A, E-D B A, M E-D B A$, Unstable ONUs

\section{INTRODUCTION}

In the recent years, the optical access networks dominate the backbone access network space. A passive optical network (PON) uses point-to-multipoint (P2MP) fiber to the premises technology in which a passive optical splitter is used to enable a single optical fiber to serve multiple subscribers premises [1-2]. The PON effectively reduces the requirement of fiber deployment and transceivers at the optical line terminal (OLT) compared to the point-to-point (P2P) optical network architectures. The PON is a form of fiber optic access network which only uses passive components such as fiber, splices and splitters from the OLT to the users' premises to minimize the network power consumptions [3]. Nowadays, the PON is being considered as a promising solution for the next generation broadband access networks due to its convergence capability of multiple service providers in it.

In the upstream direction, PON is a multipoint-to-point (MP2P) technology. Therefore, a single upstream channel is shared by the multiple optical network units (ONUs). That is why, in the time division multiple access (TDMA) PON, a dynamic bandwidth allocation (DBA) algorithm plays a significant role for providing efficient and fair utilization of the upstream channel bandwidth. The DBA is a technique by which bandwidth in a single channel can be allocated to the ONUs of a PON on the basis of their demands [1-6]. One of the main advantages of the DBA algorithms is that it can reduce the bandwidth wastage by allocating smaller transmission windows if there is lightly loaded ONUs.

A lot of DBA algorithms have been proposed by several researchers to improve the quality of services (QoSs) of PON. However, no DBA algorithm except the early DBA (E-DBA) algorithm [7] has been proposed that concern about the performance improvement of the unstable ONUs in the PON. If an ONU receives unnatural data packets and miss the usual polling instant to send Report message in a time cycle then it is called unstable ONU. The E-DBA algorithm is followed by the prediction based fair excessive bandwidth allocation (PFEBA) scheme that reduces the waiting time periods of the unstable ONUs than that of the traditional DBA algorithms. This algorithm maintains an unstable 
degree list of the ONUs in the PON by analyzing the historical data. If there is one or multiple unstable ONUs in a time cycle then the bandwidth to the unstable ONUs are allocated at the end of the time cycle, i.e., after the end of transmissions from all the stable ONUs of a time cycle. The main drawback of the E-DBA algorithm is that the unstable ONUs must need to be waited until the end of a time cycle that increases the waiting times to transmit the data packets of the queue and end to end packet delays of the unstable ONUs.

This paper presents a new DBA algorithm which is the modified version of the E-DBA algorithm called modified E-DBA (ME-DBA) algorithm. Our proposed algorithm has following characteristics. First, the proposed ME-DBA algorithm is applied for a PON consisting of $N$ ONUs and these entire ONUs are divided into $n$ groups of ONUs where each group consists of $N / n$ ONUs. Among the $N / n$ ONUs in a group, one or multiple ONUs may be unstable while the rest of the ONUs are considered as stable. Second, the better QoS provisioning is achieved for the unstable ONUs in the network by reducing the waiting time of data transmission. The performance of the proposed ME-DBA algorithm is numerically analyzed in terms of waiting time, absolute delay variations and average packet delay vs offered loads for different cycle times, i.e., different lengths of time cycles. Compared to the existing E-DBA algorithm, the proposed ME-DBA algorithm provides less waiting time, lower average delay, and lower absolute delay for any value of the cycle time.

The rest of this paper is organized as follows. Related works on different DBA algorithms are presented in the section II. The proposed ME-DBA algorithm with its timing and Gate message scheduling diagrams are explained in the section III. In section IV, we explain the packet delay, waiting time, and absolute delay variation analyses. Section V presents the simulation environment and results. Finally, the paper has been concluded in section VI.

\section{RELATED WORKS}

In this section, the existing E-DBA and different conventional DBA algorithms for the PON have been discussed.

In [2-4], the authors have proposed adaptive DBA algorithms for the PON based hybrid fiber to the home (FTTH) and wireless sensor networks (WSNs). The main concern of these adaptive DBA algorithms is to mitigate the problems associated for converging data packets of both the FTTH and WSNs in a single PON having different packet lengths and data rates. However, these adaptive DBA algorithms have not considered any unstable ONUs in the network.

The limited bandwidth allocation (LBA) algorithm is the most popular and traditional DBA algorithm that provides bandwidth allocation in the upstream channel without any prediction of data traffic in the following time cycle [8]. In the LBA algorithm, the allocated bandwidth of each ONU is upper bounded by the maximum bandwidth of $B_{\max }$. When the reported queue is less than the $B_{\max }$ the OLT grants the requested bandwidth otherwise the $B_{\max }$ is granted. The main drawback of the LBA algorithm is that it doesn't grant the bandwidth to an ONU larger than the $B_{\max }$ even the other ONUs are lightly loaded and have excessive bandwidth.

In the prediction-based DBA algorithm, the packet delay is decreased by allocating larger bandwidth to the heavily loaded ONUs. The predictive schemes are used to update the measured and predicted aggregated traffics which are employed to meet the QoSs requirements. To avoid over- or underestimation, accurate traffic predictor is required which will result in longer packet delay and degrade the network performance. In the credit-based bandwidth allocation (CBA) algorithm, when the OLT allocates the upstream bandwidth it takes some precedent transmitted frames and adds a credit to the granted bandwidth of each ONU. Its main purpose is to grant the requested window plus a credit that is proportional to the requested window size of an ONU. For this, some packets of the current time cycle do not have to be waited for transmission in the next time cycle. As a result, the average packet delay can be reduced [9].

The DBA algorithm with multiple services (DBAM) is also a prediction-based LBA algorithm. In this algorithm, the prediction is made according to the linear estimation credit where the OLT allocates the bandwidth for multiple services among the ONUs according to each bandwidth request and the service level agreement (SLA) [10].

The DBA-1 scheme works according to the priority of the window. Based on the requested window size of each ONU, all the ONUs in the PON are divided into two groups, i.e., lightly loaded ONUs 
and heavily loaded ONUs. The total bandwidth saved from the lightly loaded ONUs is proportionally re-allocated to the heavily loaded ONUs according to the requested bandwidth. This provides statistical bandwidth multiplexing among the ONUs and achieves higher link utilization [11].

In the DBA-1, a major amount of the bandwidth is wasted as idle. For solving this problem a modified DBA (M-DBA) scheme is proposed [9]. In every transmission cycle, the M-DBA guarantees a minimum bandwidth for each ONU and offers a statistical bandwidth multiplexing among the ONUs of the network to accommodate non-uniform traffic. In a word, it provides the measurement of extra bandwidth as well as guaranties a minimum bandwidth for each ONUs.

The early DBA (E-DBA) algorithm is incorporated and followed by the prediction based fair excessive bandwidth allocation (PFEBA) scheme [7]. The E-DBA algorithm can reduce the packet delay by early execution of the DBA algorithm so that the idle period is reduced and it is more effective than those of the traditional DBA algorithms. However, this E-DBA algorithm does not reduce the end-to-end packet delay and waiting time of the unstable ONUs because, in this scheme, the data packets and control messages of entire unstable ONUs in a time cycle are transmitted at the end of that time cycle.

The proposed ME-DBA scheme is the modification of this E-DBA scheme where the entire ONUs in the network is divided into $n$ groups. If there is any unstable ONU in a group then data transmission of that unstable ONU is performed at the end of that group instead of at the end of the whole time cycle.

\section{Principle of the Proposed ME-DBA Scheme}

In this section, we explain about the PON structure with unstable ONU and Gate and Report messages transmission of the existing E-DBA and the proposed ME-DBA schemes with necessary data transmission diagrams.

\subsection{Network Architecture of PON with Data Transmission in the Proposed ME-DBA Scheme}

The tree topology based PON is a very simple and efficient backbone network structure. Figure 1 shows a tree topology based PON structure with $N$ ONUs, one OLT, and upstream data transmission [2]. The OLT acts as a central office $(\mathrm{CO})$ and ONUs are directly connected to the users. Here, among the $N$ ONUs $N-1$ ONUs are considered as stable ONUs and one ONU, i.e., ONU $i$, is considered as an unstable ONU.

\subsection{Gate and Report Messages Transmission in the Existing E-DBA Scheme}

In the existing E-DBA scheme, a tree topology based PON with one OLT and $N$ ONUs are considered. Figure2(a) shows the data transmission diagram between $N$ ONUs and an OLT using control messages, i.e., Report (R) and Gate $(\mathrm{G})$. In this figure, for simplicity, only ONU $i$ is considered as an unstable ONU while rest of the $N-1$ ONUs are considered as stable ONUs. According to the E-DBA algorithm, in a time cycle, the Report messages of the entire stable ONUs are transmitted $1^{\text {st }}$ and then the Report message of the unstable ONU $i$ is transmitted.

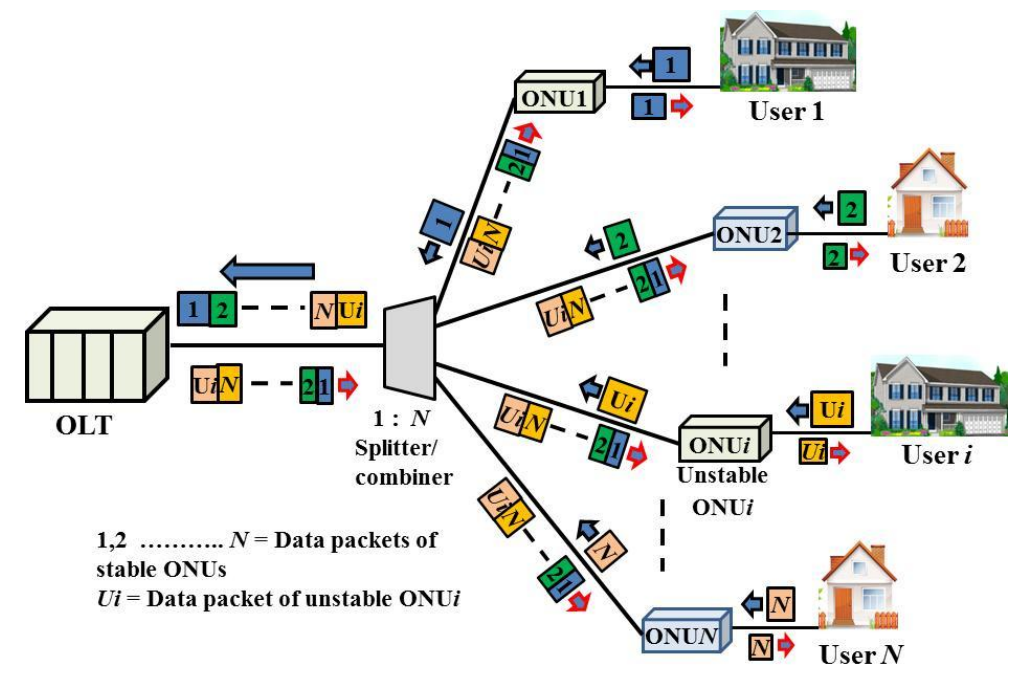

Figure1. PON Architecture with the proposed ME-DBA scheme. 


\subsection{Gate and Report Messages Transmission in the Proposed ME-DBA Scheme}

In the proposed ME-DBA scheme, a tree topology based PON with one OLT connected to the 16 ONUs are considered. Then, the entire 16 ONUs are divided into $n$ groups of ONUs where ONU $i$ is considered as an unstable ONU and other $N-1$ ONUs are considered as stable ONUs. However, both the position and number of unstable ONUs in a group of a time cycle can be random. For simplicity, only one ONU in the $1^{\text {st }}$ group, i.e., ONU $i$, is considered as an unstable ONU while rest of the ONUs in the network are stable. Figure2 (b) shows the Gate and Report messages transmission diagram between the OLT and both the stable and unstable ONUs in a time cycle based on the proposed MEDBA algorithm.

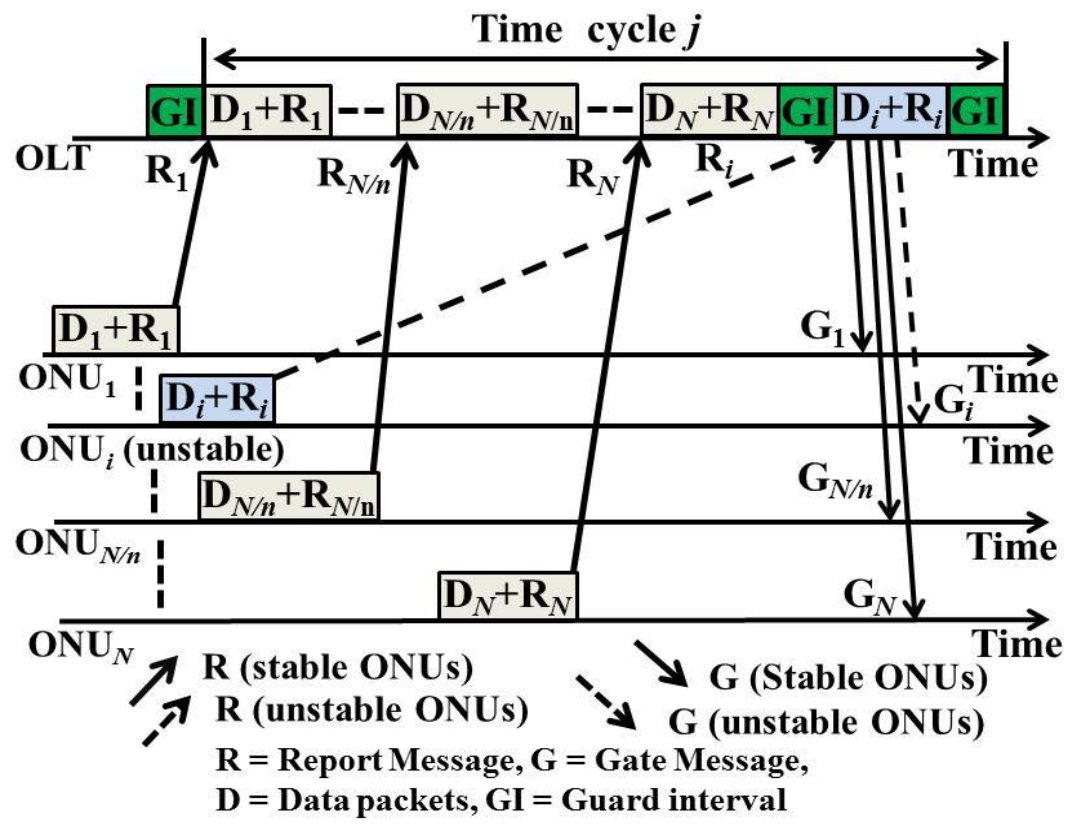

(a)

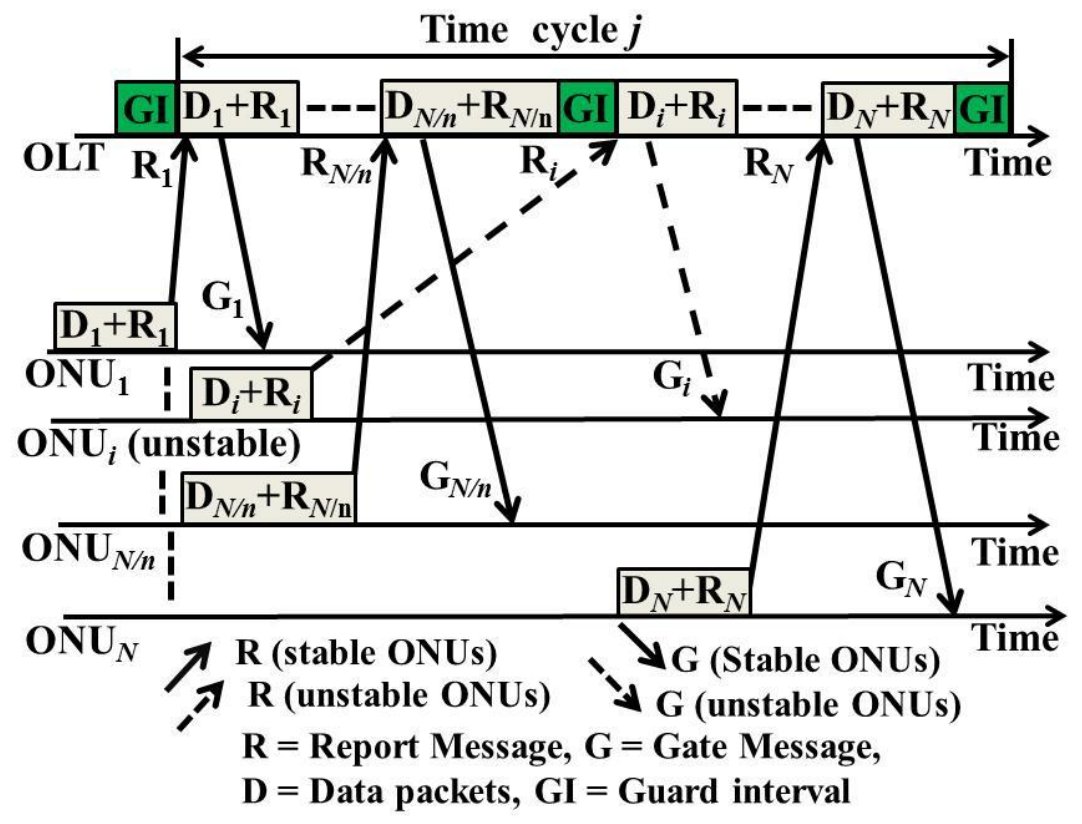

(b)

Figure2. Data transmission diagram between OLT and ONUs, (a) Existing E-DBA scheme, and (b) Proposed $M E-D B A$ scheme.

In the proposed ME-DBA algorithm, at first, all the ONUs in the network is divided into $n$ groups of ONU. If there is any unstable ONU in the $1^{\text {st }}$ ONU group, i.e, ONU1 to ONU $N / n$, then the Report message of that unstable ONU will be transmitted after the transmissions of the Report messages from all the stable ONUs of the $1^{\text {st }}$ ONU group. In the Figure2 (b), we used ONU $i$ as an unstable ONU 
while the rest of the $N / n-1$ ONUs were considered as stable ONUs. Therefore, according to the MEDBA algorithm all the stable ONUs of the $1^{\text {st }}$ ONUs group sends the Report message to the OLT. Then the Report message of the unstable ONU $i$ is transmitted. Here, in the Figure2(b), the solid lines arrows of the upward direction indicate the Report messages from the stable ONUs, i.e., $R_{1}, R_{N / n}$, and $\mathrm{R}_{N}$, while the dotted line arrow of the upward direction indicates the Report message from the unstable ONU, i.e., $R_{i}$. In contrast, the solid lines arrows of the down ward direction indicate the Gate messages from the OLT to the stable ONUs while the dotted line arrow of the downward direction indicates the Gate message from the OLT to the unstable ONU. After completing the transmissions of these Report and Gate messages between the OLT and $1^{\text {st }}$ ONUs group the next ONUs group will repeat the process.

\subsection{Gate Message Scheduling Algorithm in the Proposed ME-DBA Scheme}

In any DBA algorithm of the PON, the Gate message scheduling algorithm is essential because it provides timing synchronization and collision free upstream data transmission from the multiple ONUs through a single upstream channel. Figure 3 shows a diagram of the Gate messages scheduling algorithm that consist of a single unstable ONU. According to the principle of interleaved polling algorithm the starting Gate message can be sent from any of the ONUs because the transmission sequence of a Gate message to an ONU depends on its round trip time (RTT). Following for mulasare used to schedule the Gate messages in the proposed ME-DBA algorithm.

$$
\begin{aligned}
& T G_{i, j}=T G_{i-1, j}+\left(R T T_{i-1}+T_{G D}\right)+T_{G I}+T_{D, i-1}-\left(R T T_{i}+T_{G D}\right) \\
& T G_{N / n, j}=T G_{N / n-1, j}+\left(T_{T O}+T_{G D}\right)+T_{G I}+T_{D, N / n-1}-\left(R T T_{N / n}+T_{G D}\right)
\end{aligned}
$$

where, $T G_{i, j}$ and $T G_{N / n, j}$ are the time epochs to transmit the Gate messages to the ONU $i$ and the last ONU $N / n$ of an ONU group, respectively, at the time cycle $j, T_{G I}$ is the guard interval, $T_{G D}$ is the Gate starting delay, and $T_{T O}$ is the time out time to transmit a Gate message from an unstable ONU of an ONU group in the proposed ME-DBA scheme of PON system.

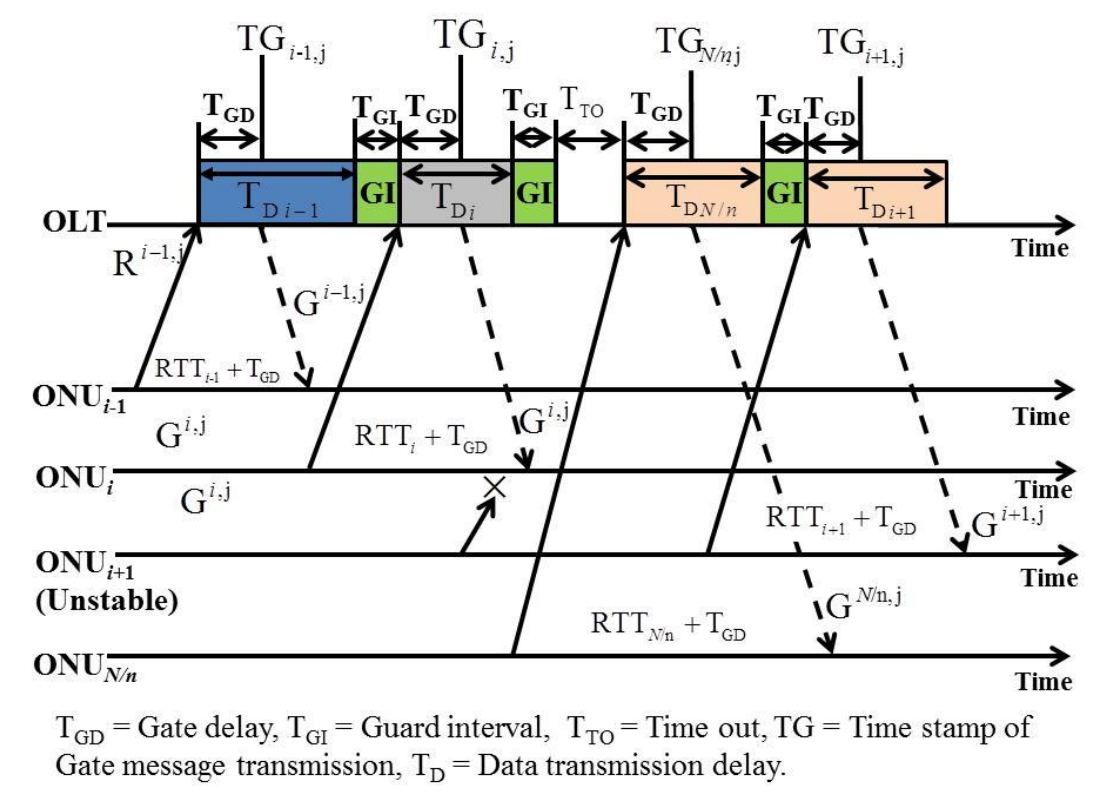

Figure3. Gate message scheduling diagram in the proposed ME-DBA scheme.

\section{Analysis of Packet delay, Waiting Timeand Absolute Delay Variation in THE PROPOSED ME-DBA SCHEME}

In this section, we explain about different performance parameters in terms of average packet delay, waiting of an unstable ONU to transmit its upstream data and average absolute delay variation in the proposed ME-DBA scheme.

\subsection{Average Packet Delay Calculations}

To calculate the average packet delay $N$ ONUs are considered in the network. According to the MEDBA scheme, total $N$ ONUs are divided into $n$ groups of ONUs and each group consists of $N / n$ 
ONUs. Calculation process of the average end to end packet delay $D_{\text {avg }}$ of the all unstable ONUs in the network is shown in equations (3) and (4).

$$
\begin{aligned}
& D_{G}=\sum_{j=i+1}^{N / n}\left(T_{G I}+T_{E O, j}+T_{R, j}+\frac{B_{j}^{G r a n t}}{R_{U}}+T_{c n g}\right) \\
& D_{a v g}=\frac{1}{N}\left(T_{a v g}^{q}+\sum_{k=1}^{n} D_{G}^{k}\right)
\end{aligned}
$$

where, $D_{G}$ is the average delay of a group, $T_{G I}$ is the length of a guard interval, $T_{E O}$ is the length of an Ethernet overhead, $T_{R}$ is the length of a Report message, $R_{U}$ is the upstream data rate, $B_{j}^{\text {Grant }}$ is the granted upstream transmission window in a time cycle, $T_{c n g}$ is the congestion time and $T_{\text {avg }}^{q}$ is the average queuing delay.

\subsection{Calculation of Waiting Time for the Unstable ONUs}

In the mathematical theory of probability, offered load is a concept in the queuing theory. The offered load is the measurement of traffic in the queue. The capacity of any data network can be defined as the amount of traffic that can be transferred through a unit of time. In the proposed ME-DBA scheme, if the $1^{\text {st }} \mathrm{ONU}$ of an ONUs group is unstable then the maximum waiting time can be summation of the transmission times of the $N / n-1$ stable ONUs. However, the waiting time of an unstable ONU randomly varies depending upon the position of it in each ONU group. The calculation of average waiting time $W_{\text {avg }}$ for the entire unstable ONUs in the PON is shown in equation (5).

$$
W_{\text {avg }}=\frac{1}{n} \sum_{k=1}^{n}\left(\sum_{j=i+1}^{N / n}\left(\frac{D_{(N / n-j)}+R_{(N / n-j)}}{R_{u}}\right)\right)
$$

where, $D$ is the data packets of an ONU in a time cycle, $R$ is the report message, and $R_{U}$ is the upstream data rate.

\subsection{Analysis of Absolute Delay Variation of an Unstable ONU}

Absolute delay variation is the inherent property of any DBA algorithm of a PON system. However, it is desire to reduce the absolute delay variations. Following equations are used to analyze the absolute delay variations in the proposed ME-DBA scheme of a PON system.

$$
\begin{aligned}
D_{A b s, j} & =\sum_{j=i+1}^{N / n}\left(T_{G I}+T_{E O, j}+T_{R, j}+\frac{B_{j}^{G r a n t}}{R_{U}}\right) \\
\Delta D_{A b s} & =\frac{1}{m} \sum_{j=1}^{m}\left|D_{A b s, j}-D_{A b s,(j+1)}\right|
\end{aligned}
$$

where, $D_{A b s, j}$ is the absolute delay in a time cycle $j, \Delta D_{A b s}$ is the absolute delay variation and $m$ is the integer, i.e., number of iterations.

\section{Simulation Results}

In this section, performance of the proposed ME-DBA scheme is evaluated by using laboratory made computer simulation programs. The performance of the proposed scheme is also compared to those of the existing E-DBA scheme in terms of waiting time, average end to end packet delay, and absolute delay variation of the unstable ONUs for different lengths of time cycles and offered loads. The system model was setup for tree topology based PON architecture with one OLT and 16 ONUs. The distances between the OLT and ONUs were considered as a random manner from 10 to $20 \mathrm{~km}$. The transmission speeds for both the upstream and downstream channels were at $1 \mathrm{Gbps}$. The Ethernet overhead was used of 576 bits and the Report message of 506 bits [12]. The maximum numbers of packets generated in a time cycle was 10 [13]. The offered load means the total bytes generated in an ONU in a time cycle. For simplicity, in the simulation, the 4 groups, i.e., value of $n$, of ONUs were used and in each group 0 to $1 \mathrm{ONU}$ was randomly considered as unstable ONU. All the parameters are shown in the Table I. 
Table1. Simulation Scenario

\begin{tabular}{|l|l|}
\hline Quantity & Values \\
\hline No. of ONUs “ $N$ ” & 16 \\
\hline No. of OLT & 01 \\
\hline No. of ONU groups & 04 \\
\hline Upstream and downstream speeds & $1 \mathrm{Gbps}$ \\
\hline Distances between OLT to ONUs & 10 to $20 \mathrm{~km}$ \\
\hline Ethernate overhead & 576 bits \\
\hline Length of a Report message & 506 bits \\
\hline Guard time & $05 \mu \mathrm{s}$ \\
\hline Maximum lengths of time cycle & 1 \\
\hline Maximum No. of packets & 10 to 2.5s \\
\hline
\end{tabular}

Figure4 compares the average delay vs length of time cycles between the proposed ME-DBA and existing E-DBA algorithms. The average end to end delay for the unstable ONUs of the existing EDBA scheme is much higher than that of the proposed ME-DBA algorithm. The delay differences between the proposed and existing DBA algorithms are increased with the length of time cycle. At the time cycle length of $2.5 \mathrm{~s}$, the average end to end delay of the existing E-DBA scheme is almost doubles than that of the proposed ME-DBA scheme.

Figure 5 compares the average waiting time of unstable ONUs between the existing and proposed DBA algorithms. From the Figure5, it is clear that if the lengths of the time cycles are increased then the average waiting times of the unstable ONUs are also increased. If the number of unstable ONUs is more, then the average waiting time will also be higher for both the existing and proposed DBA schemes. Similar to the average packet delay the existing E-DBA scheme also requires almost double waiting time than the proposed ME-DBA scheme.

The comparison of waiting time vs. offered loads for length of a time cycle of 1s is shown in Figure6. Here, the maximum offered load is 10 packets. From the results, it is confirmed that the proposed ME-DBA scheme provides lower waiting time at any value of offered load than the existing E-DBA scheme for both the cases of maximum and average waiting times.

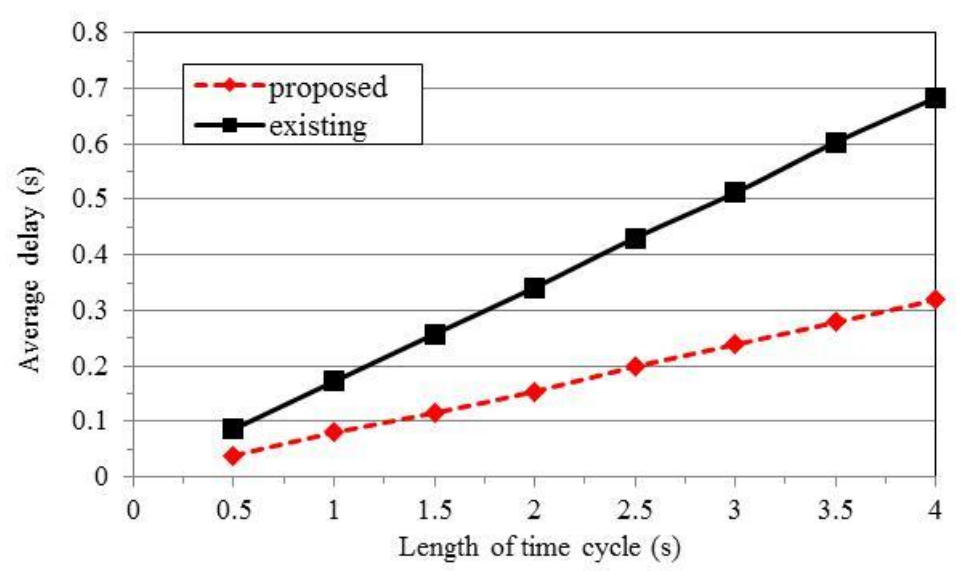

Figure4. Average delay of unstable ONUs vs. different lengths of time cycles.

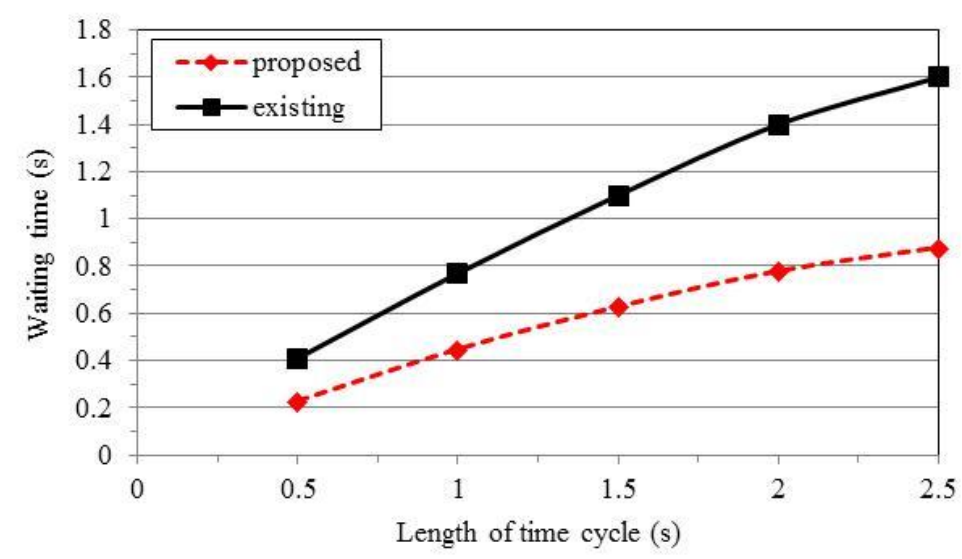

Figure5. Average waiting time of unstable ONUs vs. different lengths of time cycles. 


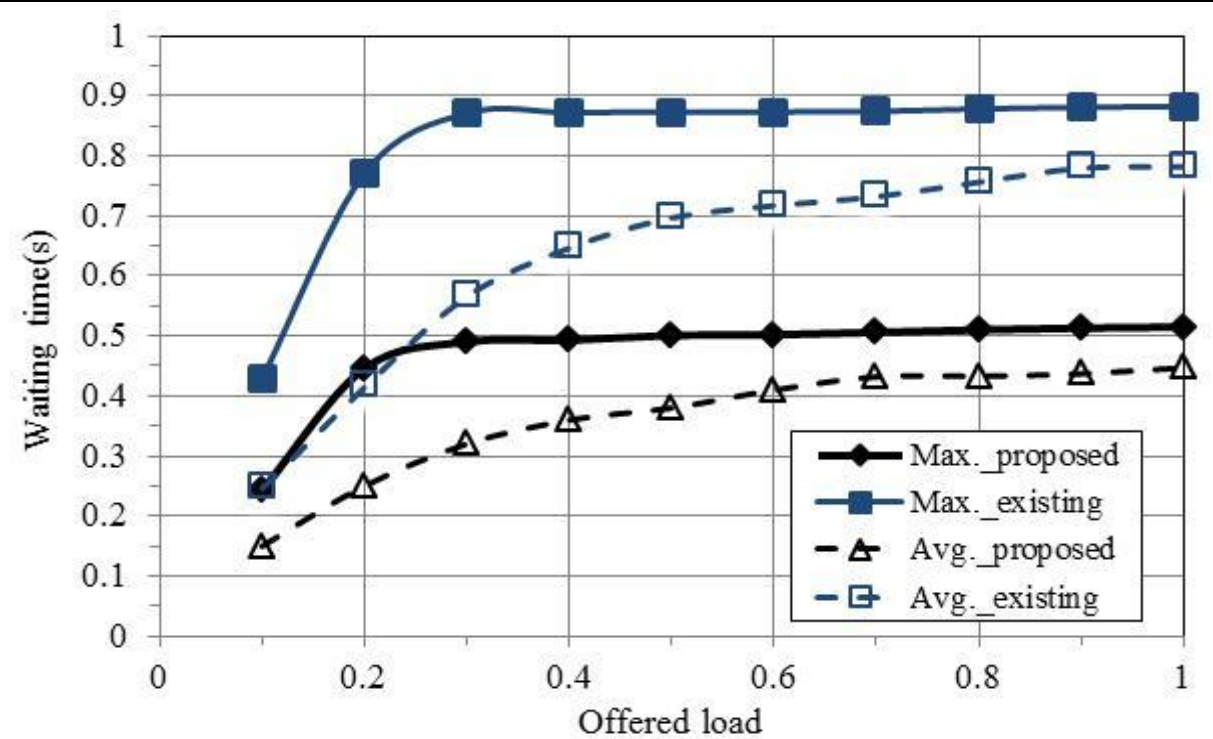

Figure6. Waiting time vs. offered loads for the length of time cycle of $1 \mathrm{~s}$.

Figures 7 and 8show the comparisons of the maximum and average waiting times of the proposed ME-DBA and existing E-DBA algorithms in term of different offered loads and lengths of time cycles. From the analysis of all these results it is also clear that the proposed scheme provides consistently lower waiting time for the unstable ONUs for any lengths of time cycles.

We have evaluated the absolute delay for the unstable ONUs of the proposed ME-DBA scheme and also have compared it with that of the existing E-DBA scheme. Figure 9 shows the instantaneous value of absolute delay for different time cycles of 1 to 500. From the figure, it is clear that the proposed scheme provides very lower absolute delay than the existing scheme over the entire range of time cycles.

Figure 10 shows the comparison of absolute delay variation vs offered load for 2.0 s time cycle. This result shows that the proposed scheme provides the maximum absolute delay variation of $0.025 \mathrm{~s}$ at the highest offered load of 1.0. In contrast, the existing E-DBA scheme provides the maximum absolute delay variation of $0.065 \mathrm{~s}$ at the highest offered load of 1.0. From this comparative analysis of absolute delay variations we can conclude that the proposed ME-DBA scheme provides better absolute delay variation performance than the existing scheme from very lower value of offered load to the highest offered load.

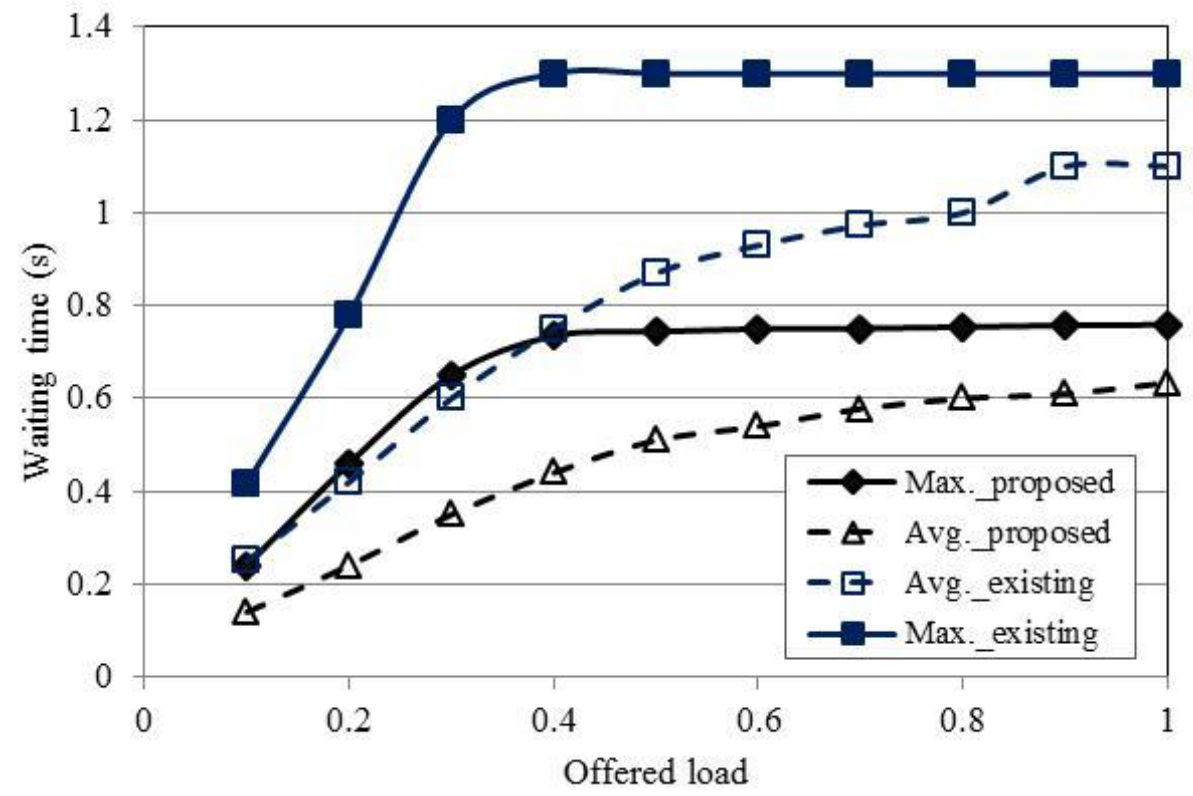

Figure7. Waiting time vs. offered loads for the length of time cycle of 1.5s. 


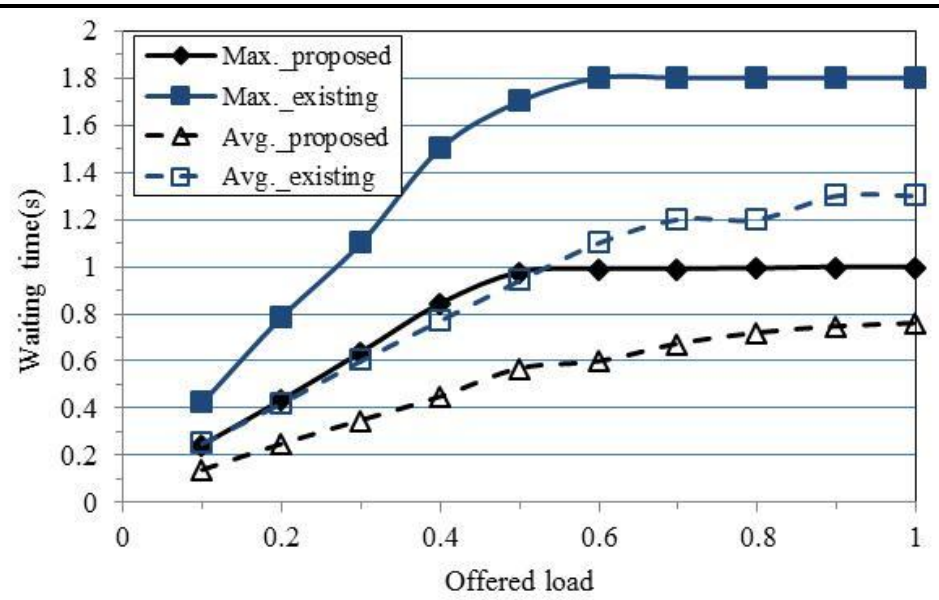

Figure8. Waiting time vs. offered loads for the length of time cycle of $2 \mathrm{~s}$.

By analyzing all these results it is confirmed that the proposed ME-DBA algorithm reduces the average end-to-end packet delay, waiting time, and absolute delay variations for the unstable ONUs than those of the existing E-DBA algorithm. The main reason is that in the proposed scheme the unstable ONUs does not need to be waited until at the end of the time cycle to transmit the data packets in the queues of unstable ONUs. In this paper, all the results have been considered for 4 groups of ONUs and for PON of 16 ONUs, i.e., each ONU group consists of 4 ONUs. If the ONU group is reduced to 2 then each group will consist of 8 ONUs in the PON of 16 ONUs and in this case the unstable ONUs will suffer from more delay. In contrast, if the ONU group is increase to 8 then each group will consist of 2 ONUs in the PON of 16 ONUs and in this case the end to end packet delay of unstable ONUs will be reduced but the processing complexity will be increased. So a tradeoff analysis between the processing complexity and delay performance is required to decide the optimum number of ONU groups in the proposed ME-DBA scheme.

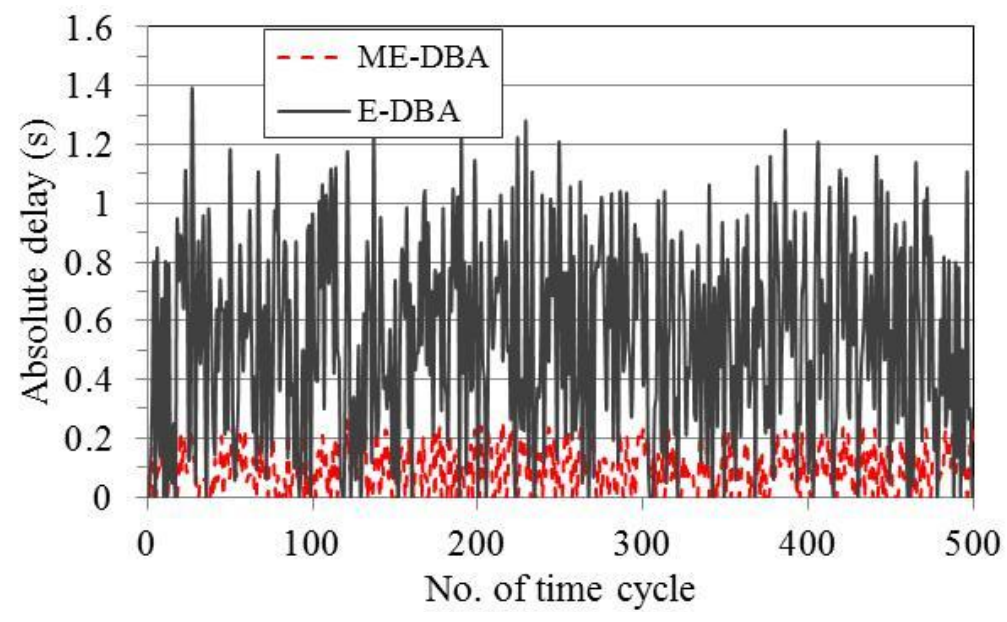

Figure9. Comparison of absolute delay for different time cycles.

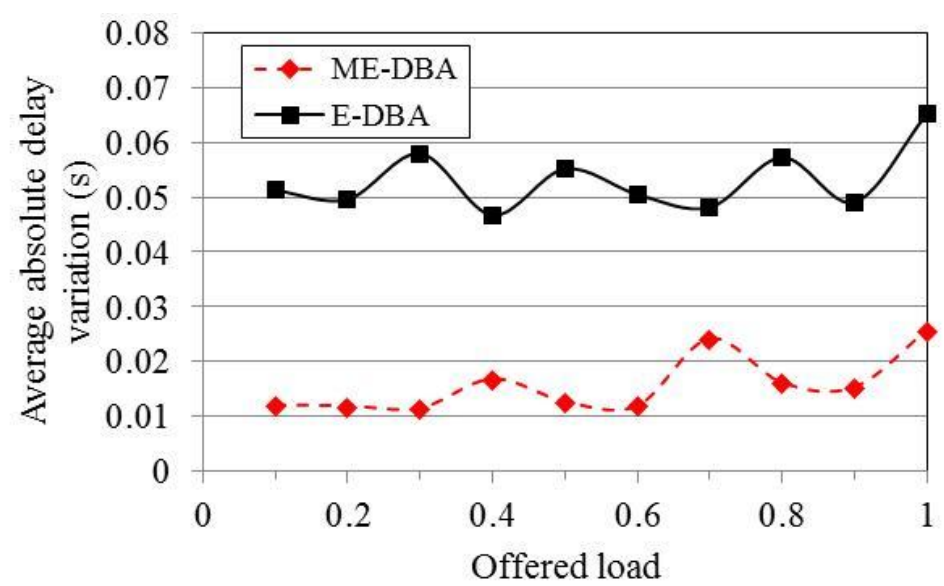

Figure10. Comparison of average absolute delay variation. 


\section{CONCLUSions}

In this paper, we have proposed a new DBA algorithm called ME-DBA to reduce the end-to-end packet delay as well as average waiting time before transmitting the data packets of the unstable ONUs. We have evaluated the average and maximum waiting times for the unstable ONUs vs various offered loads for different lengths of time cycles. From the comparison of the average packet delay to the existing E-DBA scheme it clear that the proposed ME-DBA scheme provides almost half of the average packet delay for any lengths of time cycles for the unstable ONUs. Moreover, the proposed scheme provides almost 50\% less waiting time for the unstable ONUs than the existing E-DBA scheme for any value of offered loads and lengths of the time cycles. We also have analyzed and compared the absolute delay variation between the existing E-DBA and proposed ME-DBA schemes. In terms of absolute delay variation, the proposed scheme provides $160 \%$ less delay variation than the existing scheme at the offered load of 1.0. All the simulation results significantly prove that the proposed ME-DBA scheme is better than the existing E-DBA scheme.

\section{ACKNOWLEDGEMENT}

Initial version of this paper was published in the $5^{\text {th }}$ International Conference on Informatics, Electronics and Vision (ICIEV2016), University of Dhaka, Dhaka, Bangladesh, May 13-14, 2016 [14].

\section{REFERENCES}

[1] http://en.wikipedia.org/wiki/Dynamic_bandwidth_allocation.

[2] M.Hossen, and M.Hanawa, "Dynamic bandwidth allocation algorithm with proper guard time management over multi-OLT PON-based hybrid FTTH and wireless sensor networks," IEEE/OSA Journal of Optical Communication and Networking (JOCN), vol. 5, no. 7, pp. 802812, Jul. 2013.

[3] M. Hossen, and M. Hanawa, "Adaptive limited dynamic bandwidth allocation scheme to improve bandwidth sharing efficiency in hybrid PON combining FTTH and wireless sensor networks" IEICE Transaction on Communication, Vol. E96-B, no.1, pp.127-134, Jan. 2013.

[4] M. Hossen and M. Hanawa, "Adaptive limited DBA algorithm for multi-OLT PON-based FTTH and wireless sensor networks" inProc. of the Asia Pacific Conference on Communication (APCC2012), pp. 372- 377, Jeju Island, Korea, Oct. 2012.

[5] J.Chen, L. Wosinska, M. N.Chughtai, and M. Forzati, "Scalable passive optical network architecture for reliable service delivery,"IEEE/OSA Journal of Optical Communications and Networking (JOCN), vol. 3, no. 9, pp. 667-673, 2011.

[6] W. You, "Randomized dynamic bandwidth allocation algorithm for upstream access in OFDMA-PON,"IEEE/OSA Journal of Optical Communications and Networking (JOCN), vol. 7, no.6, pp. 597-601, 2015.

[7] I. Hwang, Z. Shyu, L. Y. Ke, and C. Cheng, "A novel early DBA mechanism with predictionbased fair excessive bandwidth allocation scheme in EPON," Journal of Computer Communication, vol.31, no.9, pp. 1814-1823, Jun. 2008.

[8] G. Kramer, and B. Mukherjee, "IPACT: A dynamic protocol for Ethernet PON (EPON)," IEEE Communication Magazine, vol. 40, no. 2, pp. 74-80, Feb. 2002.

[9] Z. Peng, "A novel dynamic bandwidth allocation algorithm for Ethernet PON," M. Eng. Thesis, School of Electrical and Computer Engineering RMIT University, Australia, Mar. 2011.

[10] I. Hwang, Z. Shyu, and K. Huang, "Fuzzy logic embedded in prediction-based DBA for differentiated services on EPONs," Tamkang Journal of Science and Engineering, Vol. 12, No. 1, pp. 73-84, Jan. 2009.

[11] N. A. M. Radzi, N. M. Din, M. H. Al-Mansoori, S. K. Sadon, "Recent dynamic bandwidth allocation algorithm methods in Ethernet passive optical network," International Journal of New Computer Architectures and their Applications (IJNCAA), vol. 4, no. 4, pp. 167-176, 2014.

[12] S. Basu, M. Hossen, M. Arefeen, and B. Kundu, "An efficient multi-OLT and multi wavelengths passive optical network for differentiated classes of services," In Proc. of International Conference on Electrical Engineering and Information \& Communication Technology (iCEEiCT), Jahangirnagar University, Dhaka, Bangladesh, May, 2015. 
[13] M. Hossen, M. Hanawa, "A novel dynamic bandwidth allocation algorithm for multi-OLT and multi-wavelength PON-based hybrid networks" In Proc. of International Forum on Strategic Technology (IFOST), Cox's Bazar, Bangladesh, Oct. 2014.

[14] B.Kundu, M.Hossen, S. Basu, and M. I.Arefeen, "An algorithm for reduction of packet delay and waiting time for unstable ONUs in PON," In Proc. of $5^{\text {th }}$ International Conference on Informatics, Electronics and Vision (ICIEV2016), University of Dhaka, Dhaka, Bangladesh, May 13-14, 2016.

\section{AUTHORS' BIOGRAPHY}

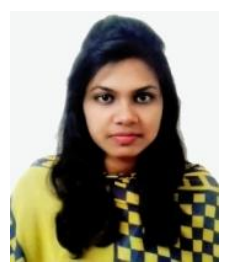

Bipashakundu, received her B.Sc. degree in Electronics and Communication Engineering from Khulna University of Engineering \& Technology (KUET), Khulna, Bangladesh, in 2015. She is working as a pre-Sales Engineer with ESL. Her research interest includes passive optical Network, Signal Processing with particular emphasis on analysis of optical fiber signal propagation.

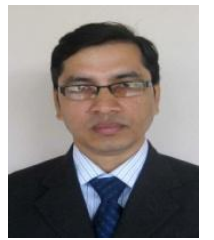

Dr. MonirHossen, received B.Sc. degree in Electrical and Electronic Engineering from Khulna University of Engineering \&Technology (KUET), Bangladesh in 2002. He completed his M.Sc. in Electronics Engineering at Kookm in University, Korea in 2010. He received Ph.D. from University of Yamanashi, Japan in 2014. Currently, he is working as an associate professor in the Department of Electronics and Communication Engineering. His present research focuses on DBA algorithm of PON, PON-based hybrid networks and their bandwidth allocation algorithms.

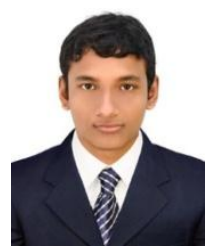

Sujit Basu, was born in Bangladesh in 1992. He received the B.Sc. Engineering degree in Electronics and Communication Engineering from Khulna University of Engineering \& Technology, Khulna, Bangladesh in July 2015.He joined Liz Fashion as Jr. System Analyst in ERP Department, now working in Bangla Trac Communication Limited as an Engineer in SOC. His research interests are passive optical network, open access network, and Networking.

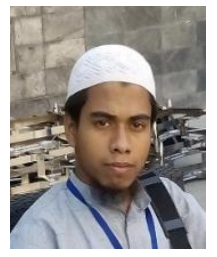

Md. ImamulArefeen, He was born in Bangladesh in 1992. He received the B.Sc. Engineering degree in Electronics and Communication Engineering from Khulna University of Engineering \& Technology, Khulna, Bangladesh in 2015.He joined Secondary Education Sector Investment Program (SESIP) under the Ministry of Education, Bangladesh, 2016 as an assistant programmer. His research interests are passive optical network, open access network, broadband power line communication etc. He is a member of the Institute of Engineers, Bangladesh (IEB). 